\title{
Special issue on unconventional cluster architectures and applications
}

\author{
Federico Silla $\cdot$ Holger Fröning
}

Published online: 9 July 2013

(C) Springer Science+Business Media New York 2013

Cluster computing has gone a long way, and provided many contributions to science and industry. Important scientific contributions have been made possible by the use of the aggregated computing power of such clusters, for instance has cluster computing helped to verify the existence of dark matter back in the 1990s. Today, the vast majority of supercomputers are based on cluster architectures. Actually, High Performance Computing (HPC) based on clusters is pervasive today, and almost every product in its design phase has gone through a work flow based on HPC. Clusters provide a huge number of computing cores, starting with a few 100s but up to several millions, which are interconnected and work collaboratively on a single problem. As the need for more computational power is steadily increasing, the interest in faster clusters persists. Even as we are currently approaching the Exascale era (Exascale provides $2^{18}$ floating point operations per second), an end of this hunger for more computing power is still not in sight.

In the beginning, clusters were unconventional architectures themselves, architected and used by few passionate scientists. During the evolution of clusters, many new methods and techniques have shown up as unconventional components, turned out to be viable and got adopted by the majority of clusters. Examples for such methods, techniques or components are user-level communication, GPUs as accelerators, Put/Get semantics, virtualization software and many more. One can clearly see that, without unconventional ap-

F. Silla

Universitat Politècnica de València, València, Spain

e-mail: fsilla@disca.upv.es

H. Fröning ( $\varangle)$

Ruprecht-Karls University of Heidelberg, Heidelberg, Germany

e-mail: froening@uni-hd.de proaches, cluster computing today would not be as it is, maybe wouldn't even exist at all.

The goal of this special issue is to provide an overview of current unconventional approaches. Even if they show inferior performance today, their potential of being a disruptive technology can turn them into viable solutions tomorrow. We present a collection of unconventional architectures and applications, trying to opening design spaces and fostering ideas about other unconventional systems. A competitive selection process was set up, with an outcome of 7 high quality papers out of 28 submissions.

The selection of articles includes work on architectures, applications and methods. With regard to architectures, we have included work on a cluster architecture based solely on FPGAs with very disruptive characteristics and an article on a low-cost, low-power cluster based on embedded ARM processing units. Application examples include a simulation of sedimentary basins on hybrid CPU-GPU clusters and an approach to reduce communication in large sparse linear system solvers. Last, the contributions that concentrate on unconventional methods include work on a direct communication path between GPUs and FPGA devices, reverse computation as an approach to fault-tolerant computing on very large cluster platforms, and finally an approach to map the well-known Map-Reduce programming model to GPU clusters.

We'd like to thank all authors, reviewers and the staff at Springer for their contributions and help. Also, we hope you'll find these articles inspiring and that they will lead to new-hopefully unconventional-ideas.

Guest Editors 